\title{
ANTICOMMONS AND OPTIMAL PATENT POLICY IN A MODEL OF SEQUENTIAL INNOVATION*
}

\author{
GASTÓN LLANES ${ }^{\dagger}$ AND STEFANO TRENTO ${ }^{\S}$
}

\begin{abstract}
When innovation is sequential, the development of new products depends on the access to previous discoveries. As a consequence the patent system affects both the revenues and the cost of the innovator. We construct a model of sequential innovation in which an innovator uses $n$ patented inputs in R\&D to invent a new product. We ask three questions: (i) what is the net effect of patents on innovation as technologies become more complex ( $n$ increases)? (ii) are patent pools welfare enhancing? (iii) what is the optimal response of patent policy as technological complexity increases? We find that the answers to these questions depend on the degree of complementarity and substitutability between the inputs used in research.
\end{abstract}

Keywords: Anticommons, Sequential Innovation, Patent Pools, Optimal Patent Policy, Complementary Monopoly, Cross-Licensing.

JEL Classification: O31, O34, L41, D4.

\footnotetext{
* We are grateful to our advisor Michele Boldrin. We thank David Levine for useful comments and suggestions on an earlier draft. We thank participants of seminars at Universidad Carlos III de Madrid and Washington University in St. Louis. All remaining errors are our responsibility. We acknowledge support from the Ministry of Education of Spain (Gastón Llanes) and the Community of Madrid (StefanoTrento).

† Departamento de Economía, Universidad Carlos III de Madrid, Calle Madrid 126, 28903 Getafe, Spain. Email: gllanes@eco.uc3m.es

$\S$ Departamento de Economía, Universidad Carlos III de Madrid, Calle Madrid 126, 28903 Getafe, Spain. Email: strento@eco.uc3m.es
} 


\section{INTRODUCTION.}

Knowledge builds upon previous knowledge. This is true for most innovations nowadays, especially in hi-tech industries like molecular biology, plant biotechnology, semiconductors and software. In some cases, the innovation consists of an improvement of an older version of the same good. In other cases, the research leading to the discovery of the new good depends on the access to research tools, techniques and inputs which are previous innovations themselves.

In any case, innovation activity will in general depend on the access to previous innovations. Depending on the structure of the patent system, many of these inventions will be protected by patents. This means that patents affect not only the revenues of the innovator, but also the cost of performing an innovation.

Recent concern has arisen on the possibility that patents (or other kinds of Intellectual Property) can restrict access to research inputs, hindering innovation as a consequence. The innovator and the owners of patents on previous inventions share the revenues of the innovation. As the number of inputs needed in research increases, the innovator faces a patent thicket and is threatened by the possibility of hold-up, namely the risk that a useful innovation is not developed because of lack of agreement with the patent holders. This problem has been dubbed the tragedy of the anticommons (Heller 1998, Heller and Eisenberg 1998). When too many agents have exclusion rights over the use of a common resource, this resource tends to be underutilized, in clear duality with the tragedy of the commons in which too many agents hold rights of use and the resource tends to be overused.

This problem may be particularly acute in biomedical research, where there is a deep controversy over the patenting of gene fragments and research tools. Take for example the case of the MSP1 antigen (Plasmodium Falciparum Merozoite Specific Protein 1), widely recognized as the most promising candidate for an anti-malarial vaccine. A study of the Commission on Intellectual Property Rights (2002) found more than 39 patent families covering DNA fragments, methods for processing fragments, production systems, vaccine delivery systems, etc. As a consequence, a potential innovator willing to commercialize a vaccine based on MSP1 must get prior permission from the owners of these property rights.

Anticommons can arise in biotechnology as well. A good example is Golden Rice, which required payment of up to 40 licenses, depending on the country of commercialization (Graff, Cullen, Bradford, Zilberman, and Bennett 2003). 
As a final example, consider the case of software patents, which cover mathematical algorithms and techniques. Software programs have become so complex that any single program may use thousands of algorithms (Garfinkel, Stallman, and Kapor 1991), possibly infringing a large number of patents. This explains the expected increase in patent litigation in this sector in the next years (think of Microsoft vs. the programmers and users of Linux), and the formation of a Patent Commons by firms involved in the Open Source community (IBM, HP, Novel, Sun, etc.).

These concerns have led the USPTO (US Patents and Trademarks Office) to recommend Patent Pools as a possible solution to limited access to biotechnology research tools (Clark, Piccolo, Stanton, and Tyson 2000). A Patent Pool is a cooperative agreement among patent holders, through which they agree on the licensing terms of a subset of their patents.

We address these issues by constructing a model of sequential innovation in which an innovator uses $n$ patented inputs to develop a new invention. Substitutability between the inputs goes from zero (perfect complements) to infinity (perfect substitutes) and the input sellers compete in prices but do not know the exact value of the innovation for the innovator.

We study how the probability of performing the innovation changes as technologies become more complex and find that it decreases when the inputs are market complements and increases when they are market substitutes. Therefore, we prove that the anticommons hypothesis may hold when inputs are essential and not easy to substitute.

Then we analyze the limiting economy when $n \rightarrow \infty$. We show that the probability of innovation is always less than socially optimal unless the inputs are perfect substitutes. Moreover, the probability of innovation goes to zero when the elasticity of substitution is below a threshold level which is higher than 1.

We also analyze the creation of a Patent Pool as a possible solution to the tragedy of the anticommons. The Patent Pool reduces the cost of innovation if the inputs are complements and increases it if the inputs are substitutes. The reason is that when the inputs are complements, an increase in the price of one of them decreases the profit of the rest of firms. The pool takes this effect into account when maximizing total profits, and therefore will set a lower price for the inputs.

Finally, we find that the optimal degree of patent protection is decreasing in $n$ if the inputs are highly complementary (i.e. have low substitutability) and increasing in $n$ in the opposite case. This surprising result contrasts with the increase in the strength of Intellectual Property Rights on research tools in the last two decades, and can be related to an extended belief that patents are good for innovation and the rent-seeking activities of agents with vested interests. 
1.1. Related literature. This paper is related to the literatures of sequential innovation, complementary monopoly and patent pools. However, we will see that the focus of this paper is different. We analyze the effects of patents on the pricing of research inputs and examine the consequences of an increase in the complexity of innovation (measured by $n$ ) on the probability that a new good is introduced and the optimal patent policy.

There is an extended literature on Sequential Innovation (Scotchmer 1991, Green and Scotchmer 1995, Chang 1995, Scotchmer 1996), which is mainly concerned with the optimal division of profits between successive innovators. Generally, in these models, there are two innovations which have to be introduced sequentially (the second innovation cannot be introduced until the first one has), and the objective is to find the patent policy that maximizes the incentives to invest in both innovations. In this paper we generalize these models by assuming that any innovation is based on $n$ of previous innovations, as in Boldrin and Levine (2005).

In this sense our paper is more related to the literature on complementary monopoly initiated by Cournot (1838). Cournot modeled a competitive producer of brass who must buy zinc and copper from two separate monopolists (zinc and copper are perfect complements), and showed that (i) the price of the inputs is higher than the price that a single provider would set, (ii) the total cost of the inputs is increasing in the number of inputs, (iii) in the limit, as $n \rightarrow \infty$ the cost of the inputs is such that the demand for the final good is zero.

Cournot's theory of complementary monopoly has been later extended in various directions. Bergstrom (1978) allows for a more general technology and studies the behavior of the factor market in depth. He is concerned with analyzing the duality between price and quantity competition and assumes a zero marginal cost of the inputs. Our model is similar to Bergstrom's, but we focus on a different problem (sequential innovation) and assume a positive marginal cost. As we will show, Bergstrom's assumption of zero marginal cost is not trivial, as the results depend both quantitatively and qualitatively on this assumption. Chari and Jones (2000) relate complementary monopoly to the externality problem. They show that, because agents play strategically, the market outcome in economies with complementarities is inefficient. This is true even if property rights are fully assigned. They also show that the most inefficient outcomes result from economies with a large number of agents, which is related to the case when the probability of innovation goes to zero as $n \rightarrow \infty$ in our model.

Cournot's theory has been also used by the literature on patent pools. Shapiro (2001) was the first to suggest that patent pools may be anticompetitive when they are formed by substitute patents, and pro-competitive when formed by complementary patents. Lerner and 
Tirole (2004) build a model to generalize Shapiro's results. They base their definition of substitutability on the shape of the payoff function of the innovator and prove that the higher the substitutability among patents, the higher the probability that the patent pool is anticompetitive. There are two main differences with respect to our model. First, in addition to showing that patent pools reduce the price of the inputs when they are complements, we also show that the pool price is independent on the number of inputs, and therefore patent pools can potentially prevent the tragedy of the anticommons from happening. Second, we base our definition of complements and substitutes on the traditional cross-price derivatives, which allows us to be more precise in determining the effects of patent pools on innovation and to explain the economic intuition behind the results.

\section{THE MODEL.}

There are $n$ research inputs $\left(x_{1}, \ldots, x_{n}\right)$ and a potential innovator who can use the $n$ inputs in $R \& D$ in order to invent a new good. The $n$ inputs have already been invented and are ready to be produced. The structure of Intellectual Property Rights is such that each input is protected by a patent, granting its owner a monopoly over it. Each patent is owned by a different patentee and thus each of the $n$ inputs is supplied by a different producer. Given that the inputs are imperfect substitutes of each other, the factor market is a differentiated goods oligopoly. The input sellers compete in prices and the value of the innovation is private information of the innovator.

2.1. Technology. The innovator can perform R\&D to invent a new final good according to the following CES technology:

$$
y=A\left(\sum_{i=1}^{n} x_{i}^{\rho}\right)^{\frac{1}{\rho}}
$$

where $y$ is a measure of the $\mathrm{R} \& \mathrm{D}$ effort, $A$ is a scale parameter, $x_{i}$ is the amount of input $i$ used, $n$ is the number of inputs and $\rho \in(-\infty, 1]$ is a technological parameter related to the substitutability between inputs.

The innovator faces an indivisibility problem, meaning that a minimum amount of $\mathrm{R} \& \mathrm{D}$ effort is required to invent a new good. When the $R \& D$ effort is below that threshold level there is no innovation. Without loss of generality we can set the threshold level at 1 , so that the indicator function for the innovation is:

$$
I=\left\{\begin{array}{l}
1 \text { if } y \geq 1 \\
0 \quad \text { otherwise }
\end{array}\right.
$$

We set the scale parameter $A$ equal to $n^{(\rho-1) / \rho}$ in order to eliminate any returns from specialization or division of labor. Usually CES production functions exhibit a property called increasing returns to 
specialization (or love for variety in the case of utility functions). Following an argument similar to Romer (1987), suppose that the production function is $y=\left(\sum_{i=1}^{n} x_{i}{ }^{\rho}\right)^{1 / \rho}$, and let $X$ be the total quantity of inputs used in production. Because of symmetry, all inputs will be used in the same quantity in the equilibrium, so $x_{i}=X / n$ for all $i$, and output will be equal to $y=n^{(1-\rho) / \rho} X$. There are positive returns to specialization because an increase in $n$ holding $X$ constant causes output to increase. We eliminate this effect by introducing $A=n^{(\rho-1) / \rho}$ in the production function.

The complexity of the innovation is measured by $n$. More complex technologies use a larger number of components or require more research tools in order to be developed. Each input is produced with a constant marginal cost of $\varepsilon>0$. We assume that the resources used to produce inputs are sold in a competitive market, so that the private and the social cost of producing inputs coincide. The assumption of no returns to specialization guarantees that the social cost of performing the innovation does not change as technologies become more complex. In other words, there is no technological advantage or disadvantage from increases in $n$.

\subsection{Value of the innovation and structure of the information.}

The social value of the innovation $(v)$ is the total surplus generated by the new product. To focus on the factor market, we will assume that the innovator is a perfect price discriminator in the final good market. This means that the private value for the innovator coincides with the social value of the innovation.

The value of the innovation $v$ is private information of the innovator. This may be because the innovator has better information about the characteristics of the new product or about the valuation of the consumers. The sellers of inputs only know that $v$ has a cumulative distribution $F(v)$. Therefore $F(v)$ is the probability that the innovation has a return less or equal than $v$. In Section 5 we show that the assumptions of perfect price discrimination and asymmetric information can be relaxed without altering the results.

The hazard function is defined as $h(v)=f(v) /(1-F(v))$, where $f(v)$ is the density function corresponding to $F(v)$. In order to guarantee the quasi-concavity of the maximization problem of the input producers, the following assumption will hold throughout the paper:

Assumption 1 (Nondecreasing hazard function). $h(v)>0$ and $h^{\prime}(v) \geq$ 0 on a support $[\underline{v}, \bar{v}]$, and $h(v)=0$ outside of this support.

This assumption on the hazard function is very general, and holds for most continuous distribution functions. We will analyze the meaning of the hazard function in section 3.1. Notice that we are not restricting $\underline{v}$ nor $\bar{v}$ to be of finite value. 
An important assumption is that the distribution of values of the innovation does not change with $n$. This assumption, together with the absence of returns to specialization in the $R \& D$ technology imply the following lemma:

Lemma 1. The probability that an invention is socially optimal does not depend on its complexity.

Proof. The probability that an innovation is socially optimal is the probability that its social value is larger or equal than its social cost. The social cost of an innovation coincides with the resources used to produce it. Therefore, the probability that an innovation is socially optimal is $\operatorname{Prob}\left(v-\sum_{i=1}^{n} \varepsilon x_{i} \geq 0\right)$. Because of the symmetry in the innovation technology, $x_{i}=1 / n$, so this probability becomes $1-F(\varepsilon)$, which depends on the distribution of social values of the innovation and the marginal cost of the inputs but not on the number of inputs used in R\&D.

In this paper, we are interested in studying the effects of increasing technological complexity on the probability of innovation. Lemma 1 assures that a change in $n$ affects this probability only through a change in the number of inputs that have to be negotiated, but not through a change in the social value or cost of the innovation. In other words, we want to compare innovations with different $n$ but the same net social value. In Section 5 we relax these assumptions by letting the value of the innovation be a function of $n$ and allowing returns to specialization in the R\&D technology. We find that the main results of the paper are not significantly affected by a change in these assumptions.

2.3. Market interaction. The players of the game are the $n$ input sellers and the innovator. A strategy for input seller $i$ is a choice of price for her input. A strategy for the innovator is a function $g: \mathbb{R}_{+}^{n} \times v \rightarrow$ $\mathbb{R}_{+}^{n}$, namely a demand $x_{i}$ for each input, as a function of the price of all the inputs and the realization of the value of the innovation.

The timing of the game is as follows: (i) the input producers simultaneously set the price of their inputs, (ii) Nature extracts a value $v$ from the distribution $F(v)$, and (iii) given prices, the innovator calculates the input mix that minimizes the cost of innovation and then decides whether to innovate or not.

The equilibrium concept we use is Symmetric Subgame Perfect Equilibrium (SSPE). A set of strategies $\left\{p_{i}\right\}_{i=1}^{n}, g$ is a SSPE if it is a Nash equilibrium of every subgame of the original game, and $p_{i}=p$ for all $i$.

The payoff for input producer $i$ is $x_{i}\left(p_{i}-\varepsilon\right)$ and the payoff of the innovator is $I v-\sum_{i=1}^{n} p_{i} x_{i}$. 
2.3.1. Innovator's Problem. Given input prices $\left\{p_{i}\right\}_{i=1}^{n}$, the innovator solves the following Cost Minimization Problem (CMP):

$$
\begin{array}{ll}
\qquad c=\min \sum_{i=1}^{n} p_{i} x_{i} \\
\text { s.t. } \quad n^{-\frac{1-\rho}{\rho}}\left(\sum_{i=1}^{n} x_{i}{ }^{\rho}\right)^{\frac{1}{\rho}} \geq 1
\end{array}
$$

The solution to this problem is the set of conditional factor demands $x_{i}$ (input demands conditioned on the innovation being performed) and the minimum cost of innovation $c$. Given $c$, the innovator will perform the innovation $(I=1)$ if $v \geq c$.

2.3.2. Input Seller's Problem. When setting the price the sellers of inputs do not know the realization of $v$. They only know that given $\left\{p_{i}\right\}_{i=1}^{n}$ the probability that $v \geq c$ (the probability of innovation) is $1-F(c)$. Therefore, the expected demand of input firm $i$ is $E\left(x_{i}\right)=$ $(1-F(c)) x_{i}$, and its Profit Maximization Problem (PMP) is:

$$
\max _{p_{i}} \quad \Pi_{i}=(1-F(c)) x_{i}\left(p_{i}-\varepsilon\right)
$$

where both $c$ and $x_{i}$ come from the CMP of the innovator.

\section{Equilibrium.}

In this section we solve recursively for the SSPE. Therefore, we begin by solving the Innovator's Problem (second stage of the game). The demands are those of a typical CES production function.

Proposition 1 (Solution of the Innovator's Problem). The conditional demand of input $i$ and the cost of innovation are:

$$
\begin{aligned}
x_{i} & =n^{-\frac{1}{1-\sigma}} p_{i}^{-\sigma}\left(\sum_{i=1}^{n} p_{i}{ }^{1-\sigma}\right)^{\frac{\sigma}{1-\sigma}} \\
c & =n^{-\frac{1}{1-\sigma}}\left(\sum_{i=1}^{n} p_{i}{ }^{1-\sigma}\right)^{\frac{1}{1-\sigma}}
\end{aligned}
$$

where $\sigma=1 /(1-\rho)$ is the elasticity of substitution between the inputs. The innovator will introduce the new good $(I=1)$ if $v \geq c$.

The restrictions on $\rho$ imply that the elasticity of substitution $\sigma$ goes from 0 (perfect complements) to $\infty$ (perfect substitutes).

Given $x_{i}$ and $c$, the symmetric equilibrium price $p$ solves

$$
p=\underset{p_{i} \geq \varepsilon}{\operatorname{argmax}}(1-F(c)) x_{i}\left(p_{i}-\varepsilon\right)
$$

where $c=n^{-\frac{1}{1-\sigma}}\left(p_{i}^{1-\sigma}+(n-1) p^{1-\sigma}\right)^{\frac{1}{1-\sigma}}$ and $x_{i}=n^{-1} p_{i}^{-\sigma} c^{\sigma}$. It is useful to notice that in the symmetric equilibrium $\left(p_{i}=p\right.$ for all $\left.i\right)$, 
$c=p$ and $x_{i}=1 / n$ for all $i$. Also, $p \geq \varepsilon$ in equilibrium because otherwise firms would be making negative profits and would find it profitable to deviate by setting a higher price.

Because of the nature of Nash equilibria, for any value of $n, \varepsilon$, and $\sigma<\infty$ there exists equilibria where $p$ is so high that the probability of innovation is zero (i.e. profits are zero for all input sellers) but any deviation by a single input seller is not enough to make it positive. However, these are trivial equilibria coming from the definition of Nash equilibria without any intrinsic economic value. We are interested in the existence of equilibria with a positive probability of innovation $(p<\bar{v})$.

The following proposition characterizes the solution of the first stage of the game (the Input Seller's Problem).

Proposition 2. A SSPE with positive probability of innovation $(p<\bar{v})$ exists and is unique. The equilibrium price solves

$$
(p-\varepsilon) h(p)=n-\sigma(n-1)(p-\varepsilon) / p
$$

The conditional input demand is $x=1 / n$, the cost of innovation is $c=p$ and the probability of innovation is $1-F(p)$.

Proof. The firm wants to maximize $(1-F(c)) x_{i}\left(p_{i}-\varepsilon\right)$. The derivative with respect to price is:

$$
D\left(p_{i}\right)=-f(c) \frac{\partial c}{\partial p_{i}} x_{i}\left(p_{i}-\varepsilon\right)+(1-F(c))\left(\frac{\partial x_{i}}{\partial p_{i}}\left(p_{i}-\varepsilon\right)+x_{i}\right)
$$

By Shepard's Lemma $\partial c / \partial p_{i}=x_{i}$, and by symmetry $c=p, x_{i}=1 / n$ and $\partial x_{i} / \partial p_{i}=-(n-1) \sigma /\left(n^{2} p\right)$. Therefore, the first order condition becomes:

$$
D(p)=-f(p) \frac{p-\varepsilon}{n^{2}}+(1-F(p))\left(-\frac{\sigma(n-1)(p-\varepsilon)}{n^{2} p}+\frac{1}{n}\right)
$$

Now we prove that the solution cannot be $\varepsilon$ nor $\bar{v}$ for $n<\infty$. $p=\varepsilon$ cannot be the equilibrium because $D(\varepsilon)=(1-F(\varepsilon)) / n>0$. Also, $p=\bar{v}$ cannot be the equilibrium both if $\bar{v}$ is finite or infinite. If $\bar{v}<\infty$, then $D(\bar{v})=-f(\bar{v})\left(\frac{\bar{v}-\varepsilon}{n}\right)<0$. On the other hand, $\lim _{p \rightarrow \infty} D(p)=$ $-\infty<0$. Therefore, the solution must satisfy $D(p)=0$. Multiplying $D(p)$ by $-n^{2} /(1-F(p))$ we get:

$$
h(p)(p-\varepsilon)+\sigma(n-1) \frac{p-\varepsilon}{p}-n=0
$$

We can be sure that equation (5) has exactly one solution because it is continuously increasing in $p$ by Assumption 1 , is negative when $p=\varepsilon$ and is positive when $p \rightarrow \bar{v}$ (Assumption 1 implies that $\lim _{p \rightarrow \bar{v}} h(p) p=$ $\infty$ for finite or infinite $\bar{v}$ ). Therefore, the solution exists and is unique. Rearranging terms in equation (5) we get the desired result. 
Example. We will find useful to illustrate the results with the help on an example based on the uniform distribution. This example has the advantage of providing an explicit solution for the equilibrium price. Specifically, assume that the value of the innovation $(v)$ is uniformly distributed between 0 and 1 . This means that $F(v)=v$ and $h(v)=$ $1 /(1-v)$. The equilibrium price is:

$$
p=\frac{a+\sqrt{a^{2}+4 \sigma \varepsilon(n-1) b}}{2 b}
$$

where $a=n+\varepsilon-\sigma(n-1)(1+\varepsilon)$ and $b=1+n(1-\sigma)+\sigma$. The cost of innovation is equal to the price and the probability of innovation is simply $1-p$.

3.1. The meaning of the First Order Condition. It interesting to analyze the meaning of the optimality condition. In the traditional case, when there is no uncertainty, the PMP is simply to maximize $x_{i}\left(p_{i}-\varepsilon\right)$. In this case, the optimal price solves:

$$
\frac{p_{i}-\varepsilon}{p_{i}}=\frac{1}{\eta_{x_{i}}}
$$

where $\eta_{x_{i}}$ is the price elasticity of the demand for inputs (in absolute value) and $\left(p_{i}-\varepsilon\right) / p_{i}$ is the Lerner index, which measures the gain over marginal cost as a proportion of price.

In our case, the PMP is to maximize $(1-F(c)) x_{i}\left(p_{i}-\varepsilon\right)$, so the optimal price solves:

$$
\frac{p_{i}-\varepsilon}{p_{i}}=\frac{1}{\eta_{x_{i}}+h(c) p_{i} x_{i}}
$$

where $h(c)$ is the hazard function.

Therefore, our model adds an specific term related with the probability of selling the inputs. The hazard function is $h(c)=-\frac{\partial(1-F(c))}{\partial c} \frac{1}{(1-F(c)}$, so it measures the proportional decrease in the probability of selling the inputs when total cost increases. We can interpret $1-F(c)$ as a demand for final good and $c$ as its price (read Section 5). Then, if we multiply the hazard function by $c$, we get the elasticity of the final demand in absolute value $\left(\eta_{D}=h(c) c\right)$.

This means that the optimal price in Proposition 2 solves:

$$
\frac{p_{i}-\varepsilon}{p_{i}}=\frac{1}{\eta_{x_{i}}+\eta_{D} \frac{p_{i} x_{i}}{c}}
$$

so our additional term is equal to the price-elasticity of final demand times the share of input $i$ in total expenditure. Notice that this result is very general, since we are not assuming any specific demand function for the inputs, nor any distribution for the values of the innovation. 
3.2. Elasticity of substitution. The price of the inputs and the cost of innovation in equilibrium depend on the elasticity of substitution, the complexity of the innovation and the marginal cost of the inputs. In the following subsections we will analyze the comparative statics of the above equilibrium.

Proposition 3. The cost of innovation is decreasing in $\sigma$.

Proof. Equation (5) provides an implicit function of $p$ in terms of $\sigma$. We can calculate $\partial c / \partial \sigma$ using the implicit function theorem (remember that $\mathrm{p}=\mathrm{c}$ in the symmetric equilibrium):

$$
\frac{\partial c}{\partial \sigma}=-\frac{(n-1)(p-\varepsilon) / p}{h(p)+h^{\prime}(p)(p-\varepsilon)+\sigma(n-1) \varepsilon / p^{2}}
$$

It is easy to see that this derivative is always negative (the numerator and the denominator are positive). The result follows.

Figure 1 depicts the cost of innovation (i.e. the price of the inputs) as a function of $\sigma$ for the uniform distribution and for $n=10$ and $\varepsilon=0.1$.

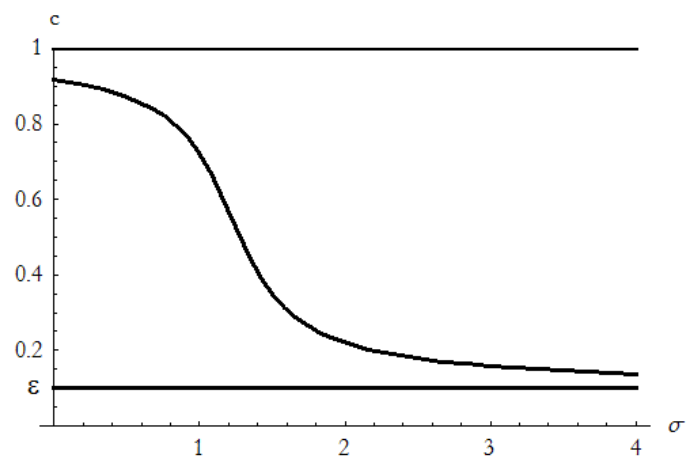

Figure 1: Cost of innovation as a function of $\sigma$.

The cost of innovation is monotonically decreasing in $\sigma$ because of increased competition as the inputs become more substitutable. As $\sigma \rightarrow \infty$ price converges to marginal cost $\varepsilon$, which is the standard Bertrand price competition result with homogeneous goods.

3.3. Complements and Substitutes. We will classify inputs in market complements and substitutes according to the sign of the cross-price derivative of expected demand which, in this setting, is equivalent to analyzing the cross-price derivative of expected profit. This classification is equivalent to the one used in game theory, where the actions of two agents are said to be complements (substitutes) when an increase in the action of one of them implies a decrease (increase) in the payoff 
of the other agent. In our model, the actions are prices and the payoff is expected profit. Notice that this is an equilibrium definition since it is based on the best response of the innovator.

Definition 1 (Market complements and substitutes). Input $j$ is a market complement (substitute) of input $i$ if $\frac{\partial E\left(x_{i}\right)}{\partial p_{j}}<0\left(\frac{\partial E\left(x_{i}\right)}{\partial p_{j}}>0\right)$.

An increase in the price of input $j$ has two effects on the expected demand of input $i$. On one hand, the conditional demand of input $i$ increases (substitution effect). On the other hand, the probability of innovation decreases because the inputs are more expensive to the innovator (innovation effect). The sign of the cross-price derivative depends on which of the two effects is stronger. The cross-price derivative is:

$$
\frac{\partial E\left(x_{i}\right)}{\partial p_{j}}=(1-F(c)) \frac{\partial x_{i}}{\partial p_{j}}+\frac{\partial(1-F(c))}{\partial p_{j}} x_{i}
$$

The first effect is related to the standard substitution effect of consumer demand theory. Remember that the Cost Minimization Problem is equivalent to the Expenditure Minimization Problem and in this case there are no wealth effects of price changes (the conditional factor demands are equivalent to Hicksian demands). In principle, the derivative $\partial x_{i} / \partial p_{j}$ could be positive, negative or zero. However, the property of negative semidefiniteness of the matrix of cross-price derivatives (which implies that every input must at least have one technical substitute), together with the symmetry of the production function, implies that this derivative is non-negative. The inputs will be technical substitutes $\left(\partial x_{i} / \partial p_{j}>0\right)$ except in the case of perfect complements, where $\partial x_{i} / \partial p_{j}=0$.

The second effect is due to the fact that the demand for innovations is downward sloping. The cost of the inputs used in research affects the profitability of innovation. Therefore, an increase in the price of any input will lower the probability of innovation. This effect is negative, except in the case of perfect substitutes, when it is zero.

Now that our definition of complementarity and substitutability is clear, we can be precise in our exposition. In what follows when we say that inputs are complements or substitutes, we mean that they are market complements or substitutes. We will see that the distinction between complements and substitutes is crucial for the predictions of the model.

The following lemma shows the value of $\sigma$ that makes the cross-price derivative equal to zero.

Lemma 2. The cross-price derivative $\partial E\left(x_{i}\right) / \partial p_{j}$ is zero in the symmetric equilibrium if and only if $\sigma=\sigma^{*}$, where $\sigma^{*}$ is the argument that solves $h\left(\frac{\sigma}{\sigma-1} \varepsilon\right)=\sigma-1$. 
Proof. The cross-price derivative is:

$$
\frac{\partial E\left(x_{i}\right)}{\partial p_{j}}=(1-F(c)) \frac{\partial x_{i}}{\partial p_{j}}-f(c) x_{i}
$$

By Shepard's Lemma, $\partial c / \partial p_{j}=x_{j}$. Imposing symmetry, $x_{i}=x_{j}=$ $1 / n$ and $\partial x_{i} / \partial p_{j}=\sigma /\left(n^{2} p\right)$. Rearranging terms we get:

$$
\frac{\partial E\left(x_{i}\right)}{\partial p_{j}}=\frac{1}{n(1-F(p))}\left(\frac{\sigma}{p}-h(p)\right)
$$

This will be zero in the symmetric equilibrium only when $h(p)=\sigma / p$. Introducing this into the first order condition (5) and rearranging we get $(p-\varepsilon) / p=\sigma^{-1}$ or $p=\sigma \varepsilon /(\sigma-1)$. Plugging this value of $p$ in $h(p)=\sigma / p$ we get the desired result.

The following proposition classifies inputs in complements and substitutes according to Definition 1. It is interesting to see that this distinction depends on the values of $\sigma$ and $\varepsilon$, but not on the value of $n$.

Proposition 4. In the symmetric equilibrium, inputs are complements when $\sigma<\sigma^{*}$ and substitutes when $\sigma>\sigma^{*}$.

Proof. We know from Lemma 2 that the cross-price derivative is zero when $\sigma=\sigma^{*}$ and that its sign depends on $\sigma / p-h(p)$. The latter expression is increasing in $\sigma$ because $p$ is decreasing in $\sigma$ from Proposition 3 and $h$ is non-decreasing in $p$ from Assumption 1. The result follows.

Interestingly, the value of $\sigma$ which divides inputs in complements and substitutes has to be larger or equal than 1 . To see this, suppose that $\sigma^{*}<1$. This means that $h\left(\varepsilon \sigma^{*} /\left(\sigma^{*}-1\right)\right)<0$, which is not possible. In the case of the uniform distribution, for example, inputs are complements when $\sigma<(1+\varepsilon) /(1-\varepsilon)$ and substitutes when $\sigma>$ $(1+\varepsilon) /(1-\varepsilon)$.

3.4. Patent Pools. Until now, the research inputs were priced noncooperatively. In this subsection we analyze what happens when all inputs are priced cooperatively, either by a collective institution such as a patent pool or by a single patent holder (monopolist) that owns all the patents. Proposition 5 shows the equilibrium price in this case. The difference with the previous case is that now the patent holder maximizes joint-profits and therefore takes into account the cross-price effects between expected demands.

Proposition 5 (Patent Pool). The equilibrium price when all the inputs are priced cooperatively $\left(p^{*}\right)$ is the argument that solves $h(p)(p-$ $\varepsilon)=1$. 
Proof. Given the symmetric input demands, the pool wants to sell a symmetric bundle. Therefore $x_{i}=1 / n$ and $p_{i}=p$ for all $i$ and the pool wants to maximize total profits $n(1-F(p))(p-\varepsilon)$. The first order condition is $n(-f(p)(p-\varepsilon)+1-F(p))=0$. Rearranging terms we get the desired result.

Notice that $p^{*}$ depends only on the functional form of $h$ and the value of $\varepsilon$, but not on the values of $\sigma$ or $n$. The following proposition compares the cost of innovation when the inputs are priced individually (c) with that of a patent pool $\left(p^{*}\right)$.

Proposition 6. The cost of innovation when the inputs are priced non-cooperatively (c) is equal to that of a patent pool $\left(p^{*}\right)$ when the cross-price derivative is zero $\left(\sigma=\sigma^{*}\right)$, it is larger when the inputs are complements $\left(\sigma<\sigma^{*}\right)$ and it is smaller when the inputs are substitutes $\left(\sigma>\sigma^{*}\right)$.

Proof. We know from the proof of Lemma 2 that when $\sigma=\sigma^{*}$, the cross-price derivative is zero and $\sigma=p h(p)$. Replacing this in (5) and rearranging we get $h(p)(p-\varepsilon)=1$, which is the cooperative result. Given that $p$ is decreasing in $\sigma$, whereas $p^{*}$ is independent of $\sigma, p>p^{*}$ when $\sigma<\sigma^{*}$ and $p<p^{*}$ when $\sigma>\sigma^{*}$.

The difference between cooperative and non-cooperative pricing is that in the first case the firms take into account the effect of an increase in the price of one input on the demand for the rest. When $\sigma=\sigma^{*}$ this effect is zero so the price of the pool coincides with that of the noncooperative equilibrium. When $\sigma<\sigma^{*}$ the effect is negative, so the pool knows that an increase in price will decrease the demand for the rest and will set a price smaller than the uncoordinated input sellers. The opposite happens when $\sigma>\sigma^{*}$.

In the case of the uniform distribution, the pool price is $p^{*}=(1+$ $\varepsilon) / 2$. Figure 2 compares this price with the non-cooperative price for $\varepsilon=0.1$ and $n=5$.

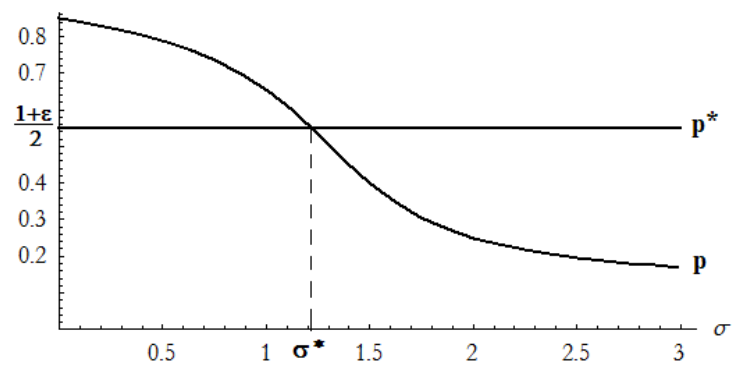

Figure 2: Cooperative and non-cooperative pricing. 
3.5. Increasing complexity. Proposition 7 shows that the sign of the effect of an increase in the complexity of the innovation $(n)$ depends on whether the inputs are complements or substitutes.

Proposition 7. The cost of innovation increases as innovation becomes more complex if the inputs are complements $\left(\sigma<\sigma^{*}\right)$ and decreases if the inputs are substitutes $\left(\sigma>\sigma^{*}\right)$.

Proof. We are looking for the effect of a unit increase in $n$, but it will suffice to determine the sign of $\partial c / \partial n$. Equation (5) provides an implicit function of $c$ in terms of $n$. Therefore, we can calculate $\partial c / \partial n$ using the implicit function theorem:

$$
\frac{\partial c}{\partial n}=\frac{1-\sigma(p-\varepsilon) / p}{h^{\prime}(p)(p-\varepsilon)+h(p)+\sigma(n-1) \varepsilon / p^{2}}
$$

We know that the denominator is always positive. Therefore, the sign of this derivative depends on the sign of the numerator.

From equation (5) we get the following relation in equilibrium $\sigma(p-$ $\varepsilon) / p=(n-h(p)(p-\varepsilon)) /(n-1)$. Introducing this in the numerator and operating, it becomes $(h(p)(p-\varepsilon)-1) /(n-1)$. We know that $h(p)(p-\varepsilon)=1$ when $\sigma=\sigma^{*}$ from the proof of Proposition 6. Given that $h(p)(p-\varepsilon)$ is increasing in $p$, it is decreasing in $\sigma$. Therefore, the numerator is positive when $\sigma<\sigma^{*}$ and it is negative when $\sigma>\sigma^{*}$. The result follows.

The probability of innovation is simply $1-F(c)$, so it moves in an opposite direction to the cost:

$$
\frac{d P r}{d n}=-f(c) \frac{d c}{d n}
$$

As before, the effect on the probability of innovation of an increase in the complexity of innovation depends on the substitutability between the inputs. If inputs are complements, then the probability decreases as $n$ increases. If inputs are substitutes, then the probability increases as $n$ increases.

Figure 3 shows what happens in the uniform distribution example as the complexity of the innovation increases from $n=5$ to $n=15$, for $\varepsilon=0.1$. The cost schedules cross when $\sigma=1.22$, which is exactly $\sigma^{*}=(1+\varepsilon) /(1-\varepsilon)$. This means that the cost of innovation increases if the inputs have low substitutability and decreases in case of high substitutability.

Proposition 7 is the most important result of the paper. It says that patents are very harmful when innovation is sequential and the research inputs are essential or difficult to substitute, but do not pose an important problem when inputs are easily replaceable.

Figure 4a shows cost as a function of $n$ for complementary inputs and $\varepsilon=0.1$ in the case of the uniform distribution. As innovation becomes more complex, the cost of innovation increases and converges 


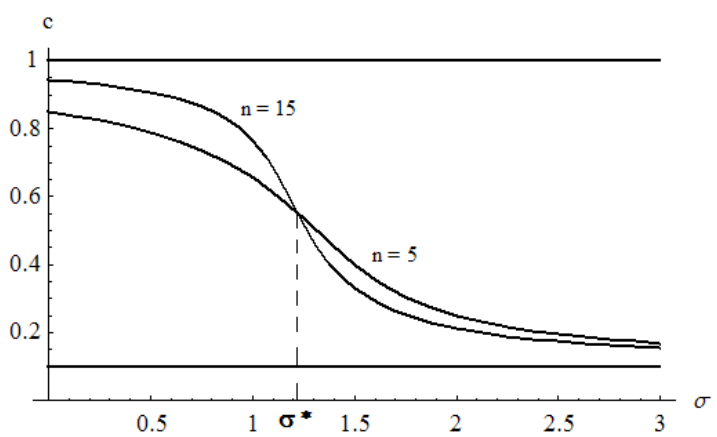

Figure 3: Effects of an increase in the complexity of innovation.

to 1 when $n \rightarrow \infty$. This means that the probability of innovation decreases with $n$ and converges to 0 . Convergence is faster when $\sigma$ gets closer to zero. When the substitutability between the inputs is very low ( $\sigma$ close to zero), the probability of innovation is very small even for simple innovations (low $n$ ).

Figure $4 \mathrm{~b}$ shows that the conclusions change when the research inputs are substitutes. In this case the cost of innovation decreases when the complexity of innovation increases (i.e. the probability of innovation increases with $n$ ).

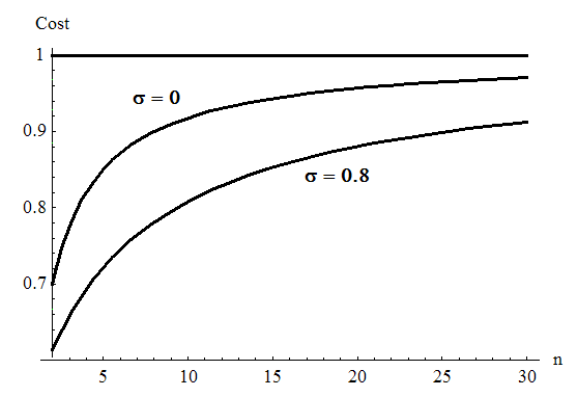

(a) Complements.

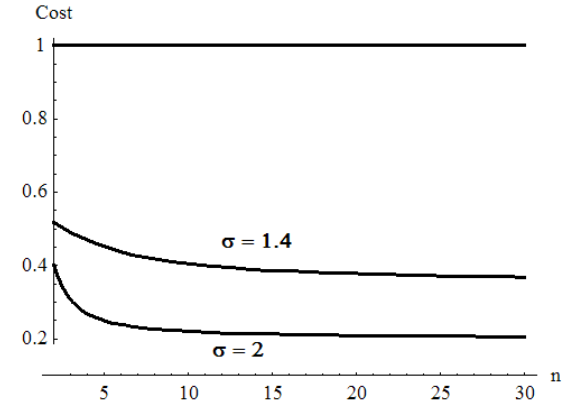

(b) Substitutes.

Figure 4: Cost of innovation as a function of $n$.

3.6. The Tragedy of the Anticommons Revisited. The model presented in this paper gives a formal treatment to the tragedy of the anticommons (Heller 1998). An anticommon arises when multiple owners have the right to exclude each other from using a scarce resource, causing its inefficient under-utilization. This problem is symmetric to the tragedy of the commons, where multiple owners have the right to 
use a scarce resource, but nobody has exclusion rights and resources tend to be overused.

In our model, the scarce resource is the net social value of the innovation $(v-\varepsilon)$ to be shared between the innovator and the patent holders. Each patent holder decides the selling price of her input. It is interesting to notice that when $\sigma \leq 1$ all the inputs are essential to perform the innovation so all the input sellers can potentially impede the innovation by setting a high price.

We show in Proposition 6 that when the inputs are market complements the cost of gathering all the inputs with fragmented property rights is larger than what it would be if there was a sole owner of all the inputs or the inputs were priced cooperatively by a collective institution like a patent pool. Moreover, according to Proposition 7, this problem gets worse as technologies become more complex, requiring more and more inputs in order to be developed. These results hold not only for perfect complementarity between the inputs, but whenever the elasticity of substitution is not sufficiently large to compensate the negative effect of price changes on the probability of innovation.

The result in Proposition 6 is consistent with the findings of Shapiro (2001) and Lerner and Tirole (2004). The difference is that since we use the standard definition of complements and substitutes, we can be more precise in explaining the reasons why the cost of gathering a bundle of patents increases or decreases with the formation of the pool. It all depends on the relation between input demands. If the joint profit of the rest of firms decreases with an increase in the price of one firm (the input is a complement of the rest of inputs), a pool will consider it optimal to set a lower price when taking this effect into account. The opposite happens when the inputs are substitutes.

3.7. High complexity and Monopolistic Competition. It is interesting to analyze the equilibrium of the economy when $n \rightarrow \infty$ for two reasons. First, $n \rightarrow \infty$ represents innovations that are highly complex and therefore require a large number of inputs to be developed. The innovator faces a patent thicket and has to gather inputs from many patentees. We know how the probability of innovation changes as $n$ increases, but it is interesting to determine in what cases it will go to 0 or $1-F(\varepsilon)$. Second, in this limiting economy there is an infinite number of input sellers, so the effect of a price change by a single firm has a infinitesimal impact on the cost of innovation, and the market becomes monopolistically competitive.

Proposition 8 characterizes equilibria with positive probability of innovation $(p<\bar{v})$. In this case there are values of $\sigma$ for which there is no equilibrium with positive probability of innovation.

Proposition 8. A SSPE with $p<\bar{v}$ exists only when $\sigma>\hat{\sigma}$ where $\hat{\sigma}=\frac{\bar{v}}{\bar{v}-\varepsilon}$. The equilibrium price and cost of innovation are $p=\frac{\sigma}{\sigma-1} \varepsilon$. 
Proof. Dividing the first order condition (5) by $n$, we get:

$$
h(p)(p-\varepsilon) \frac{1}{n}+\sigma\left(\frac{n-1}{n}\right)\left(\frac{p-\varepsilon}{p}\right)-1=0
$$

As $n \rightarrow \infty$, the term with the hazard function goes to zero. This is because each firm becomes negligible and does not affect the probability of innovation on its own. It is clear that the equilibrium price of the limiting economy solves:

$$
\sigma\left(\frac{p-\varepsilon}{p}\right)-1=0
$$

Therefore, $p=\frac{\sigma}{\sigma-1} \varepsilon$, which is between $\varepsilon$ and $\bar{v}$ only when $\sigma>\frac{\bar{v}}{\bar{v}-\varepsilon}$.

It is interesting to comment on three characteristics of the equilibrium. First, any $p \geq \bar{v}$ is an equilibrium for any value of $\sigma$ in this limiting economy. If $p \geq \bar{v}$ the probability of innovation is zero, but if a single input seller deviates, its impact on the cost of innovation is infinitesimal, so the probability of innovation (i.e. expected profits) remains unchanged. Therefore, there are no profitable deviations when $p \geq \bar{v}$.

Second, the equilibrium quantity $x_{i}$ goes to zero as $n \rightarrow \infty$. This is because the number of inputs is increasing towards infinity but the total quantity of inputs required is keeping constant, given our assumptions on the innovation technology.

Finally, it is easy to show that $1 \leq \hat{\sigma}<\sigma^{*}$. The first inequality follows trivially from the fact that $\hat{\sigma}=\bar{v} /(\bar{v}-\varepsilon)$. Therefore, $\hat{\sigma}=1$ only when $\bar{v} \rightarrow \infty$ or $\varepsilon=0$. For the second inequality, it is enough to compare the equilibrium price when $\sigma=\hat{\sigma}$ with the equilibrium price when $\sigma=\sigma^{*}$, since price is decreasing in $\sigma$. When $\sigma=\hat{\sigma}$, price is equal to $\bar{v}$. When $\sigma=\sigma^{*}$ we know that the equilibrium price solves $h(p)(p-\varepsilon)=1$. If $p=\bar{v}$, then $h(p)(p-\varepsilon) \rightarrow \infty$, which is much larger than 1. For $h(p)(p-\varepsilon)$ to decrease and approach 1, $p$ has to decrease. This means that equilibrium price is larger with $\hat{\sigma}$ and therefore $\hat{\sigma}<\sigma^{*}$.

Figure 5 shows the cost schedule as a function of $\sigma$ when $\bar{v}=1$ and $\varepsilon=0.1$. The equilibrium of the limiting economy does not depend on the distribution of $v$, but it depends on the upper bound of the support of the distribution.

The equilibrium price is the same that Dixit and Stiglitz's (1977) monopolistic competition model. When inputs are substitutes, firms set a mark-up over marginal cost equal to $1 /(\sigma-1)$. This means that the pricing inefficiency decreases as $n$ increases, but it does not disappear even when $n \rightarrow \infty$.

For complements, the outcome depends on whether $\sigma$ is greater or less than $\hat{\sigma}=\bar{v} /(\bar{v}-\varepsilon)$. When $\sigma>\bar{v} /(\bar{v}-\varepsilon)$, firms set a mark-up just like in the substitutes case. When $\sigma \leq \bar{v} /(\bar{v}-\varepsilon)$, the only equilibria have $p \geq \bar{v}$ and so the probability of innovation is zero. In this case, as 


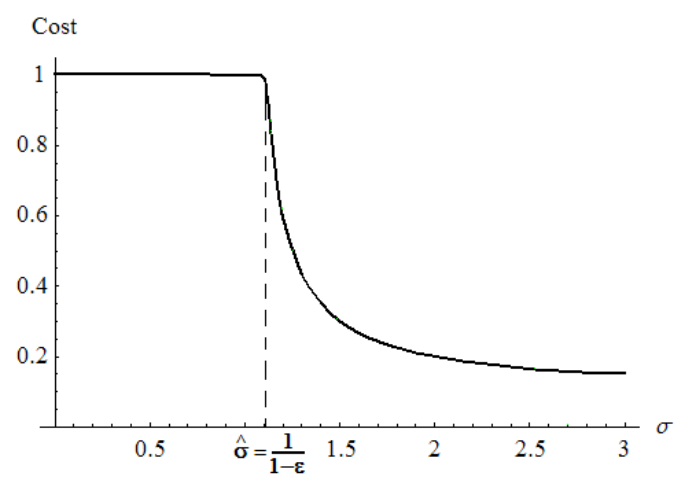

Figure 5: Cost of innovation in the limiting economy.

$n$ increases the inefficiency due to monopoly pricing increases and it is at its maximum when $n \rightarrow \infty$.

\section{Patent Policy and Welfare Analysis.}

In this section we look for the patent policy that maximizes the probability that a socially desirable innovation is performed. We will see that this policy also maximizes expected social welfare when the inputs are perfect complements or perfect substitutes.

We assume that the patent policy in the input market is related to that of the output market so that patents for research inputs cannot be removed without removing patents for final goods too. Therefore, patent policy affects not only the cost of innovation, but also the revenues from selling the new good. Although the model remains static in nature, these modifications add a dynamic flavor to it, since we can imagine that the new good that is being created will become an input for a future invention.

First of all, we have to be more precise with respect to the effect of patents in the final good sector. If the new good is protected by a patent, the innovator is the unique seller of the good and remains as a perfect price discriminator. If the new good is not protected by a patent, a large number of imitators will enter the market and perfect competition will drive price to marginal cost. In both cases the quantity of the final good produced will be optimal, so there is no inefficiency coming from monopoly pricing in the final goods market.

Patent policy will be represented by a continuous parameter $\phi \in$ $[0,1]$. Higher values of $\phi$ mean stronger patent protection.

This policy parameter $\phi$ can represent patent length. The innovator is granted a patent for a proportion $\phi$ of the useful life of the invention, but at the same time, a proportion $\phi$ of previous patents has not yet expired. $\phi$ could also be related to the novelty requirement, or to the 
strength with which IP law is enforced in courts. In this case, there is a probability $\phi$ that the innovator is granted a patent and that the patent can be defended successfully in court. At the same time, only a proportion $\phi$ of previous inventors has been granted a patent.

Under both interpretations, the revenue of the innovation is $\phi v$, and the innovator has to pay a non-competitive price $p_{i}$ for $\phi n$ inputs (covered with patents), and the competitive price $\varepsilon$ for the remaining $(1-\phi) n$ inputs.

The patent policy that maximizes the probability of innovation also maximizes expected social welfare only when the inputs are perfect complements or perfects substitutes. In the intermediate cases, the patent policy not only affects the probability of innovation but also its social cost (through its effect on the equilibrium combination of inputs). Therefore, when $0<\sigma<\infty$, the policy that maximizes the probability will not maximize expected social welfare in general.

The assumptions above guarantee that the patent regime does not affect the social value of the innovation (i.e. the total consumer surplus from the new good). In order for the patent system not to affect the social cost of the inputs either, it should lead to an optimal combination of the inputs for the innovation, which requires all inputs to be used in the same quantity. If this is not the case, the cost of the inputs used in the innovation will be larger than $\varepsilon$.

This condition holds when the inputs are perfect complements, given that conditional demands are constant and independent of price, and when the inputs are perfect substitutes, given that the price of the inputs will be equal to $\varepsilon$ independently of the patent policy. When the inputs are imperfect substitutes, on the other hand, the innovator will not use the optimal combination if the inputs have different prices, which will happen if some inputs are protected by patents and others are not $0<\phi<1$.

4.1. Perfect Complements. Given that it is not possible to obtain a general solution for the optimal policy, we will first focus in the perfect complements case $(\sigma=0)$. Then, we will show that the results depend on the substitutability between the inputs with the aid of numerical simulations.

Under perfect complementarity, the probability that revenues $(\phi v)$ exceed the cost of the innovation is $1-F(c / \phi)$ and the conditional input demands are constant and equal to $1 / n$, regardless of whether the inputs are protected by patents or not. The cost of innovation is

$$
c=\sum_{i=1}^{\phi n} \frac{1}{n} p_{i}+(1-\phi) \varepsilon
$$


The Profit Maximization Problem (PMP) of the input sellers becomes:

$$
\max (1-F(c / \phi)) \frac{1}{n}\left(p_{i}-\varepsilon\right)
$$

The following proposition characterizes the equilibrium given patent policy $\phi$ :

Proposition 9. The cost of innovation in the symmetric equilibrium solves

$$
h(c / \phi)(c-\varepsilon)=n \phi^{2}
$$

and its derivative with respect to patent policy $\phi$ is

$$
\frac{d c}{d \phi}=\frac{h^{\prime} c(c-e)+2 n \phi^{3}}{\phi\left(h^{\prime}(c-\varepsilon)+h \phi\right)}
$$

Proof. The first order condition of the input seller's PMP is:

$$
-f\left(\frac{c}{\phi}\right) \frac{1}{\phi} \frac{\partial c}{\partial p_{i}} \frac{p_{i}-\varepsilon}{n}+\left(1-F\left(\frac{c}{\phi}\right)\right) \frac{1}{n}=0
$$

It is easy to prove that in a symmetric equilibrium, $p-\varepsilon=(c-\varepsilon) / \phi$. Imposing symmetry, using Sheppard's Lemma and rearranging we get expression (9). Equation (10) follows from a simple application of the implicit function theorem.

The following lemma presents a simplification which allows us to find the optimal patent policy.

Lemma 3. The patent policy that maximizes the probability of innovation solves $\frac{\partial c}{\partial \phi}=\frac{c}{\phi}$.

Proof. The problem of maximizing $1-F(c(\phi) / \phi)$ is equivalent to the problem of minimizing $c(\phi) / \phi$, given that $F$ is non-decreasing. The first order condition for the latter is

$$
\frac{c^{\prime}(\phi) \phi-c(\phi)}{\phi^{2}}=0
$$

Rearranging this expression, we get our desired result.

Proposition 10. The optimal patent policy $\phi^{*}$ solves

$$
h\left(\frac{2 \varepsilon}{\phi}\right)=\frac{n \phi^{2}}{\varepsilon}
$$

and its derivative with respect to the complexity of the innovation is

$$
\frac{\partial \phi^{*}}{\partial n}=-\frac{\phi^{4}}{2\left(h^{\prime} \varepsilon^{2}+n \phi^{3}\right)}
$$

which is always negative. 
Proof. From Lemma 3 we know that the optimal policy solves $\frac{\partial c}{\partial \phi}=\frac{c}{\phi}$. Using (10) we have that:

$$
\frac{h^{\prime} c(c-e)+2 n \phi^{3}}{\phi\left(h^{\prime}(c-\varepsilon)+h \phi\right)}=\frac{c}{\phi}
$$

Simplifying we get:

$$
h(c / \phi) c=2 n \phi^{2}
$$

Combining this last expression with (9) we get that at the optimal policy the cost of innovation is $c=2 \varepsilon$. Introducing this in (11), we get our first result. The second result follows from an application of the implicit function theorem.

From Proposition 10 it follows that the optimal policy is larger than zero and less than one for $\varepsilon>0$. This means that some protection is always desirable, even when the research inputs cannot be substituted at all. However, the most important result in Proposition 10 is that the degree of patent protection should decrease when technologies become more complex.

4.2. Perfect Substitutes. Proposition 10 shows the effects of increases in complexity on the optimal policy when the inputs are perfect complements. However, the result is completely reversed when the inputs are perfect substitutes. In this case, the cost of innovation is $\varepsilon$ for any level of patent protection. This means that the patent policy should be directed at maximizing the revenues from the innovation, so $\phi^{*}=1$ regardless of the complexity of the innovation.

4.3. Imperfect Substitutes. A comparison of the results with $\sigma=0$ and $\sigma \rightarrow \infty$ gives an intuition of what happens for intermediate values of $\sigma$. There will be a $\bar{\sigma}$ such that the optimal policy decreases for lower degrees of substitutability, and increases in the opposite case. Given that it is not possible to get an explicit formula for $\bar{\sigma}$, we resort to a numerical analysis. Figure 6 shows the optimal patent policy as a function of $\sigma$ for $n=10$ and $n=20(\varepsilon=0.1)$. We can see that the optimal policy decreases as $n$ goes from 10 to 20 for $\sigma<1.137$, and increases in the opposite case, which certifies our previous conjecture.

\section{Extensions.}

In this section we analyze the consequences of relaxing some of the basic assumptions of the model.

5.1. Social value and cost depend on complexity. Until now, we have assumed that the distribution of values of the innovation and the social cost of the inputs do not depend on $n$, and that there are no returns from specialization. Under these assumptions, a change in $n$ only changes the number of input producers with whom the innovator 


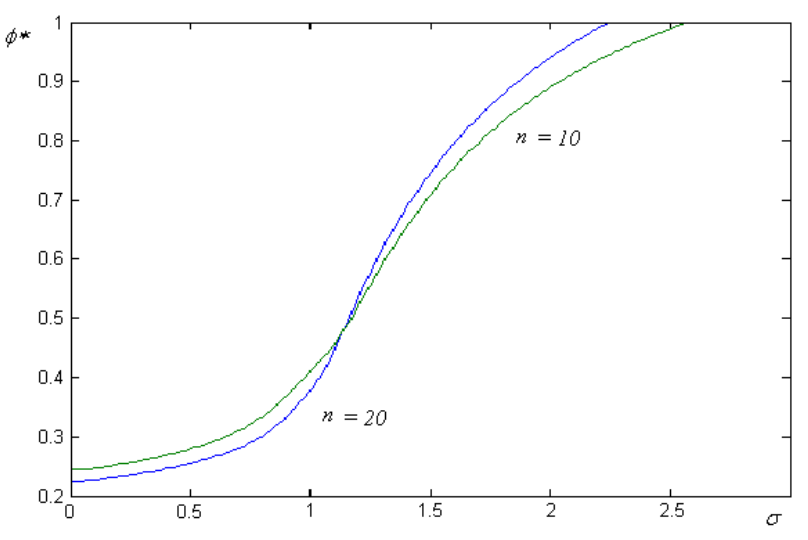

Figure 6: Optimal policy for different values of $n$.

has to negotiate patents in order to innovate, but does not change the probability that the innovation is socially valuable.

However, it could be argued that the revenues of the innovator or the cost of the inputs are increasing or decreasing in $n$, or that a higher number of inputs has a positive impact in the R\&D technology due to a higher division of labor. All these changes have equivalent effects on the probability of innovation so we will concentrate on changes in the distribution of returns of the innovation.

Let the return of the innovation be $a(n) v$, with $a^{\prime}(n) \geq 0$ or $a^{\prime}(n) \leq 0$ and $\lim _{n \rightarrow \infty} a(n)=a_{\infty}>0 . \quad v$ has a cumulative distribution $F(v)$ as before. Notice that we are not setting an upper bound on $a_{\infty}$. All we require is that if $a$ is non-increasing it does not go to zero as $n \rightarrow \infty$. This is because if $a_{\infty}=0$ then the distribution of values of the innovation will collapse to zero and the innovation will never be profitable when $n$ is very large by assumption.

The probability of innovation is $1-F(c / a)$, and in the symmetric equilibrium $c=p$ and $x=1 / n$. The equilibrium price of the inputs (i.e. the cost of innovation) solves:

$$
(p-\varepsilon) h(p / a) / a=n-\sigma(n-1)(p-\varepsilon) / p
$$

but we are more interested in the ratio $k=p / a$. Replacing in the previous equation we have:

$$
(k-\varepsilon / a) h(k)=n-\sigma(n-1)(k-\varepsilon / a) / k
$$

This equilibrium is equivalent to the one in Proposition 2, thinking of $k$ as the cost of innovation and $\varepsilon / a$ as the social cost of the inputs. We can prove the same theorems as before with respect to the difference between complements and substitutes, the welfare effects of patent pools and $\partial k / \partial \sigma$. However, $\partial k / \partial n$ will be different because now $\varepsilon / a$ is a function of $n$. 
Using the implicit function theorem on the equilibrium relation (12) we get:

$$
\frac{\partial k}{\partial n}=\frac{\frac{h(k)(k-\varepsilon / a)-1}{n-1}-\frac{n a^{\prime}}{a} \frac{\varepsilon / a}{k-\varepsilon / a}}{h^{\prime}(k)(k-\varepsilon / a)+h(k)+\sigma(n-1)(\varepsilon / a) / k^{2}}
$$

As before, the sign of this derivative depends only on the sign of the numerator, but now there is an additional term which shifts the threshold value of $\sigma$ that divides positive and negative changes in $k$. This threshold value will be to the left of $\sigma^{*}$ when $a^{\prime}(n)>0$ and to the right of $\sigma^{*}$ when $a^{\prime}(n)<0$.

Two important remarks are in order. First, if $a^{\prime}(n)$ is large then the last term in the numerator will determine the sign of the derivative. In this case, the effect of changes in $n$ on revenues completely overcomes the effect on the pricing of inputs, and $\partial k / \partial n$ has the opposite sign of $a^{\prime}(n)$ irrespective of the value of $\sigma$. Second, even for small $a^{\prime}(n)$, when $a^{\prime}(n)>0$ and $\sigma \rightarrow \infty$ the derivative is always positive. Therefore when $a^{\prime}(n)$ is small and positive, there are two regions where $\partial k / \partial n$ is positive: one with low values of $\sigma$ and another with large values of $\sigma$.

According to the previous analysis, assuming that the return of the innovation depends on $n$ has an effect on the derivative of the probability of innovation with respect to $n$. In what follows we will show that this assumption has no significant effect on the analysis of the equilibrium as $n \rightarrow \infty$.

The equilibrium price solves:

$$
h(p)(p-\varepsilon) \frac{1}{a n}+\sigma\left(\frac{n-1}{n}\right)\left(\frac{p-\varepsilon}{p}\right)-1=0
$$

When $n \rightarrow \infty$, the first term will go to zero because $a_{\infty}>0$. Therefore, the equilibrium price is the same as before, $p=\frac{\sigma}{\sigma-1} \varepsilon$, which is less than the maximum possible revenue $\left(a_{\infty} \bar{v}\right)$ only if $\sigma>a_{\infty} \bar{v} /\left(a_{\infty} \bar{v}-\varepsilon\right)$. When $\sigma \leq a_{\infty} \bar{v} /\left(a_{\infty} \bar{v}-\varepsilon\right)$, on the other hand, there is no equilibrium price such that the probability of innovation is positive.

The probability of innovation is $1-F\left(p / a_{\infty}\right)$. There are two possible cases. If $a_{\infty}<\infty$ then the probability of innovation is less than optimal, just as in the basic model. If $a_{\infty}=\infty$ then the probability of innovation will go to 1 for $\sigma>1$ and 0 for $\sigma \leq 1$, which is the same as assuming $\varepsilon=0$ in the basic model.

5.2. On the distribution of values of the innovation. Another assumption of the basic model is that there is asymmetric information on the value of the innovation (read Gallini and Wright (1990) and Bessen (2004) for good discussions of why this assumption makes sense). However, we will show that the results do not depend on the existence of asymmetric information. All that is needed for the results is a downward sloping demand for innovations. 
An alternative interpretation could be that there is a continuum of innovators with decreasing returns to their innovations. Suppose that the innovators are indexed by the return to their innovations, which ranges between $\underline{v}$ to $\bar{v}$. Now, $F(v)$ is the measure of innovations with a return less or equal than $v$. Also, assume that the innovations do not compete against each other in the final good market and that the input sellers cannot price discriminate between the innovators. It is easy to see that all the previous results translate directly into this setting. All that changes is that now $1-F(c)$ is not the probability of innovation but the measure of innovations performed.

A second alternative would be to assume that there is a continuum of perfectly competitive innovators and that the inputs are not used in research, but in the production of every unit of final good. This description is closer to Cournot's (1838) theory of complementary monopoly. The production function of output is $y=\left(\sum_{i=1}^{n} n^{\rho-1} x_{i}{ }^{\rho}\right)^{1 / \rho}$ and the demand of the final good is $y=1-F\left(p_{y}\right)$, where $y$ and $p_{y}$ are the quantity and price of the final good. In this case, the input demands in Proposition 1 still solve the CMP, but $c$ becomes a marginal cost per unit of final good. Competition leads price to marginal cost $\left(p_{y}=c\right)$, but $c$ still remains above $\varepsilon$. $1-F(c)$ is now the equilibrium quantity of final good, but all the previous results still hold. However, now there is a welfare loss from the anti-competitive pricing in the inputs market, which is approximately equal to $(c-\varepsilon)(F(c)-F(\varepsilon)) / 2$.

5.3. No price discrimination. We can also relax the assumption that the innovator is a perfect price discriminator. Dropping this assumption introduces a wedge between the social and private values of the innovation. This means that the distribution of values of innovation changes, and that now there is also an inefficiency in the final good sector. Assume that the social value of the innovation is still distributed according to $F(v)$, with probability density function $f(v)$. However, the private value of the innovation is now $v_{p}$, which is less than the social value of the innovation. With a linear demand for the final good, for example, the private return of the innovation would be $v_{p}=v / 2$, which has a probability density function given by $2 f\left(2 v_{p}\right)$. The qualitative results are the same as before. All that changes is that now the probability of innovation decreases for each value of $\sigma$, and so the values of $\sigma^{*}, \hat{\sigma}$ and $\bar{\sigma}$ increase. Also, the optimal patent protection is lower for each value of $\sigma$ and $n$ than in the case of perfect price discrimination.

5.4. Uncertain return of the innovation. We have also assumed that the innovator is the only one that knows the value of the innovation. In this section we ask what happens if $v$ is also unknown to the innovator. Formally, we do this by changing the timing of the game: (i) the input producers simultaneously set the price of their inputs, (ii) 
given prices, the innovator calculates the input mix that minimizes the cost of innovation and then decides whether to innovate or not, and (iii) Nature extracts a value $v$ for the innovation from the distribution $F(v)$.

We begin by solving the second stage of the game. The innovator decides what would be the optimal combination of inputs to perform the innovation in case he decides to perform it. This leads to the same cost of innovation and conditional demands as before. Then, the innovator decides whether to perform the innovation or not, in order to maximize expected profits $E(v)-c$. The innovation will be performed if $E(v) \geq c$ and will not be performed otherwise. If $E(v)<\varepsilon$, then the innovation will never be performed, so we assume that $E(v) \geq \varepsilon$. We also assume that the innovator will perform the innovation when indifferent between performing it or not $(E(v)=c)$.

The uncertainty has now passed from the input sellers to the input producer. The problem of the input sellers is deterministic, they know $E(v)$ and they know that if the price is higher than $E(v)$ the innovation will not be performed. In this case, the inputs are always market substitutes unless $\sigma=0$. It is easy to show that the innovation will always be performed, and that the elasticity of substitution only affects the distribution of payoffs between the input sellers and the innovator.

Lemma 4 shows that input demands are discontinuous at a certain price, and Proposition 11 proves that in the symmetric equilibrium $c \leq E(v)$ so the innovation is always performed.

Lemma 4. Input demands are discontinuous at

$$
p_{i}=\left(n E(v)^{1-\sigma}-\sum_{j \neq i} p_{j}{ }^{1-\sigma}\right)^{\frac{1}{1-\sigma}}
$$

Proof. The demand for inputs is positive if the cost of innovation is not larger than the expected value of the innovation, that is:

$$
n^{-\frac{1}{1-\sigma}}\left(\sum_{i=1}^{n} p_{i}{ }^{1-\sigma}\right)^{\frac{1}{1-\sigma}} \leq E(v)
$$

Rearranging terms, we get the condition on the price of the input:

$$
p_{i} \leq\left(n E(v)^{1-\sigma}-\sum_{j \neq i} p_{j}{ }^{1-\sigma}\right)^{\frac{1}{1-\sigma}}
$$

If $p_{i}$ is larger than this value, then the innovation is not performed and the demand for all inputs is zero.

The input sellers want to maximize profits $x_{i}\left(p_{i}-\varepsilon\right)$. Proposition 11 states the solution of the game. 
Proposition 11. The equilibrium price when the return of the innovation is uncertain for the innovator is:

$$
p= \begin{cases}\frac{\sigma(n-1)}{\sigma(n-1)-n} \varepsilon & \text { if } \sigma>\frac{n}{n-1} \frac{E(v)}{E(v)-\varepsilon} \\ E(v) & \text { otherwise }\end{cases}
$$

Proof. After imposing symmetry, the derivative of $x_{i}\left(p_{i}-\varepsilon\right)$ with respect to $p_{i}$ becomes:

$$
D(p)=\frac{1}{n}\left(-\frac{\sigma(n-1)}{n} \frac{p-\varepsilon}{p}+1\right)
$$

Lemma 4 implies than if the derivative with respect to price is positive at $p=E(v)$, this is a symmetric equilibrium, as firms are making positive profit, do not want to lower price $(D \geq 0)$, and would have a zero profit if they would rise price. This happens when $\sigma \leq \frac{n}{n-1} \frac{E(v)}{E(v)-\varepsilon}$.

When $\sigma>\frac{n}{n-1} \frac{E(v)}{E(v)-\varepsilon}$, on the other hand, the equilibrium price solves the unrestricted first order condition $D(p)=0$.

\section{Conclusions.}

Innovation in hi-tech industries is sequential (each innovation builds on previous innovations) and complex. In this context patent protection affects the expected profits of the innovator in two ways: (i) by increasing her expected revenues because of the monopoly power it grants over the innovation; (ii) by increasing the cost of innovation, since the innovator must pay each of the previous patented innovations on which her new good is built.

In this paper we constructed a model of a complex and sequential innovation to analyze how the probability that an innovation is privately profitable changes as technologies become more complex and the inputs used in research are patented. We found that the results depend on the substitutability of the research inputs.

When the inputs are complements, the profitability of the innovation is decreasing in the technological complexity. In the limit (when $n \rightarrow$ $\infty$ ), when the degree of substitutability is below a threshold level, which is higher than 1, the innovation is never profitable. This paper therefore gives a formal treatment of the tragedy of the anticommons.

On the other hand, when the inputs are substitutes, the profitability of the innovation is increasing in technological complexity. Even in this case, when $n \rightarrow \infty$, the cost of gathering all the inputs for the innovation is always too high from a social point of view and thus the probability of innovation is suboptimal.

Since we used a very general model not relying on strong assumptions, our findings generalize the results of the literature on complementary monopoly, mainly concerned with perfect complementarity.

We also studied what happens when inputs are priced cooperatively, either by a collective organization as a patent pool or by a single owner 
of all the inputs. We found that the cost of the innovation decreases with respect to the non-cooperative pricing, when inputs are market complements, while it increases when inputs are market substitutes. This result is in line with the intuition of Shapiro (2001) and the model of Lerner and Tirole (2004). In this sense the contribution of this paper has been to use a more precise definition of complementarity and substitutability. This has allowed us to study with greater detail what is the intuition behind this result.

Finally we studied the welfare implications of the patent system. We find that, when research inputs are complements, the optimal degree of patent protection is decreasing in the complexity of the innovation. This is the exact opposite of what we observe in the real world: the complexity of technology is increasing but patents are becoming stronger every day. Not only they have been recently extended to sectors previously lacking protection (sexually reproduced plants, software, business methods, products and processes of biotechnology, including plants and animals). Also patent length has been increasing over the years, and an patent systems are being created in countries where they did not previously exist. We think this is a contradiction worth to be studied further.

\section{REFERENCES}

Bergstrom, T. C. (1978): "Cournot Equilibrium in Factor Markets," Discussion paper, University of Michigan working paper.

Bessen, J. (2004): "Holdup and Licensing of Cumulative Innovations with Private Information," Economics Letters, 82(3), 321-26.

Boldrin, M., AND D. Levine (2005): "The economics of ideas and intellectual property," Proceedings of the National Academy of Sciences, 102(4), 1252-1256.

Chang, H. F. (1995): "Patent Scope, Antitrust Policy, and Cumulative Innovation," RAND Journal of Economics, 26(1), 34-57.

Chari, V., AND L. Jones (2000): "A reconsideration of the problem of social cost: Free riders and monopolists," Economic Theory, 16(1), 1-22.

Clark, J., J. Piccolo, B. Stanton, And K. Tyson (2000): "Patent pools: a solution to the problem of access in biotechnology patents?," Discussion paper, United States Patent and Trademark Office.

Commision on Intellectual Property Rights (2002): "Integrating Intellectual Property Rights and Development Policy: Report of the Commision on Intellectual Property Rights," London.

Cournot, A. (1838): Researches Into the Mathematical Principles of the Theory of Wealth. Irwin (1963).

Dixit, A. K., AND J. E. Stiglitz (1977): "Monopolistic Competition and Optimum Product Diversity," American Economic Review, 67(3), 297-308.

Gallini, N. T., And B. D. Wright (1990): "Technology Transfer under Asymmetric Information," RAND Journal of Economics, 21(1), 147-60.

Garfinkel, S., R. Stallman, And M. Kapor (1991): "Why patents are bad for software." Issues in Science and Technology, 8(1), 50-55.

Graff, G., S. Cullen, K. Bradford, D. Zilberman, And A. Bennett (2003): "The public-private structure of intellectual property ownership in agricultural biotechnology," Nature Biotechnology, 21(9), 989-995. 
Green, J. R., And S. Scotchmer (1995): "On the Division of Profit in Sequential Innovation," RAND Journal of Economics, 26(1), 20-33.

Heller, M. A. (1998): "The Tragedy of the Anticommons: Property in the Transition from Marx to Markets," Harvard Law Review, 111(3), 621-688.

Heller, M. A., AND R. S. Eisenberg (1998): "Can Patents Deter Innovation? The Anticommons in Biomedical Research," Science, 280(5364), 698-701.

Lerner, J., And J. Tirole (2004): "Efficient Patent Pools," American Economic Review, 94(3), 691-711.

Romer, P. M. (1987): "Growth Based on Increasing Returns Due to Specialization," American Economic Review, 77(2), 56-62.

Scotchmer, S. (1991): "Standing on the Shoulders of Giants: Cumulative Research and the Patent Law," Journal of Economic Perspectives, 5(1), 29-41.

(1996): "Protecting Early Innovators: Should Second-Generation Products Be Patentable?," RAND Journal of Economics, 27(2), 322-331.

Shapiro, C. (2001): "Navigating the Patent Thicket: Cross Licenses, Patent Pools, and Standard Setting," in Innovation policy and the economy, ed. by A. B. Jaffe, J. Lerner, and S. Stern, vol. 1, pp. 119-150. MIT Press for the NBER. 